Editorial

\title{
Small bite, big threat: World Health Day 2014
}

\author{
Lorenzo Savioli ${ }^{1}$ and Raman Velayudhan ${ }^{2}$
}

\section{Introduction}

This year the theme for World Health Day on 7 April is "vector-borne diseases". It provides an ideal opportunity for individuals and communities to get involved in tackling this particular group of diseases that continue to be an important public health problem. Vector-borne diseases pose a devastating obstacle to human health and overall economic development. Responsible for approximatley $17 \%$ of the estimated global burden of infectious diseases [1], vector-borne diseases contribute to lost productivity, school absenteeism, social stigma, poverty, high health-care costs and overburdened public health services [2]. Additionally over 3.5 billion people worldwide - mostly the poorest segments of populations - are also at risk.

Mosquitoes are perhaps the most commonly known disease vectors but others, including ticks, sandflies, fleas, bugs and freshwater snails, also carry a range of diseases, some of which are fatal.

\section{Impact}

Many of these "neglected" diseases thrive in poor communities in remote areas where people have poor or no access to the benefits of public health systems, safe drinking-water and basic sanitation. Combined, vector-borne diseases such as malaria, dengue, schistosomiasis, leishmaniasis, Chagas disease, lymphatic filariasis, yellow fever and onchocerciasis kill more than 1 million people annually [3]. They also leave many more with chronicillnesses, resulting in life-long disabilities, which prevent them from leading productive lives.
Of all the vector-borne diseases, dengue is the most rapidly-spreading in the world. The incidence of the disease has increased 30-fold in the past 50 years; it is now expanding from urban to rural settings with more serious outbreaks in countries and continents with no previous occurrence. This is foreseen to escalate further with climate change, mass urbanization, and movement of people and goods. Today, dengue is endemic in over 125 countries [4], with recent cases reported in Europe. Outbreaks of dengue impose a devastating burden on populations, health systems and economies; in the Americas, dengue illness is estimated to cost US\$ 2.1 billion a year [5].

\section{Challenges}

While vector control is of crucial importance for dengue and all vector-borne diseases, this intervention faces many challenges including the development of insecticide resistance among vectors, lack of trained personnel, poorly equipped facilities (laboratories and insectaries), weak entomological surveillance systems and environmental changes requiring appropriate and rapid response to evolving circumstances.

The rapid emergence of insecticide resistance in several countries is a major public health problem [6]. Resistance largely results from heavy reliance on a single class of insecticides called pyrethroids used in long-lasting insecticidal nets (LLINs) and for indoor residual spraying (IRS). Pyrethroids are not only highly effective and environmentally sound but are also the least expensive of the four classes of insecticides available for public health vector control.
LLINs and IRS remain very effective tools for malaria control. However, the gains made with their use are at risk unless urgent action is taken to prevent further development and spread of resistance among vectors. WHO therefore urges disease-endemic countries to assess the state of insecticide resistance locally and to implement comprehensive and preemptive insecticide resistance management strategies. Endemic countries are also encouraged to design a monitoring plan and build capacity to manage and interpret resistance data to give strategic direction for country programmes. In foci where vector-borne diseases overlap, programmes urgently need to share information on resistance status and implement integrated insecticide resistance management strategies. Through sound planning and management, endemic countries can delay the evolution of resistance, preserve the effectiveness of existing insecticides and even reverse resistance in some settings.

\section{Integrated vector management}

WHO promotes integrated vector management (IVM) as the best approach to strengthen vector control in a way that is compatible with national health systems [7]. Defined as a rational decision-making process to optimize the use of resources, IVM aims to achieve comprehensive control strategies in close collaboration with and support from partners in the global public health community, policy-makers and other sectors, such as agriculture and environmental health. IVM implementation involves five 
key strategies: advocacy, social mobilization and legislation, collaboration among all relevant sectors, integrated activities, evidence-based decision-making and capacity building. Sustainable programmes need enhanced knowledge on vector biology and transmission as well as technical guidelines that detail lucid operating procedures. Programmes should be well managed with efficient logistics and monitoring and evaluation frameworks.

Today, quality-assured standards for public health pesticides are being used in 96 countries where vector-borne diseases are endemic [8]. WHO has also invested significant resources in supporting its Member States to strengthen capacity for quality control of pesticides. In 2010, a WHO survey involving 142 countries endemic for or at risk of major vectorborne diseases found that ministries of health in $90 \%$ of the 113 responding countries follow WHO specifications for quality control in their requirements to procure pesticide products [8]. The World Health Assembly Resolution WHA63.26 (2010) aimed to strengthen country capacity to safely manage public health pesticides throughout their life cycle; this was further supported by the Eastern Mediterranean Region (EMR) resolution EM/RC58/R.10 (2012) on managing the use of public health pesticides, which urged Member States to develop a comprehensive pesticide policy and legislation, operate an effective pesticide registration scheme and establish or strengthen their capacity to regulate pesticides throughout their life cycle.

\section{Challenges in WHO EMR}

Countries of EMR harbour a huge proportion of the global burden of vector-borne diseases, including malaria, leishmaniasis lymphatic filariasis and emerging dengue fever resulting in the projections for disability life adjusted years in 2015 to be an estimated 1,266 818 within the Region (Health Statistics and Informatics Department, WHO, 2008).

Despite the significant progress made in eliminating malaria, in 2012 about 280 million people in nine countries in the EMR were at some risk of malaria, and an estimated 120 million were at high risk [9]. The main challenges affecting the Region include the coverage and quality of operational implementation which are constrained by insufficient expertise to manage programmes efficiently and effectively. There is a lack of adequate resources (entomological laboratories and insectaries) and skills (untrained existing and insufficient staff) to establish an entomological surveillance system for routine monitoring of key entomological indicators and qualitative monitoring of vector control operations (IRS and LLINs), which are crucial in guiding the programme on vector control interventions in different endemic settings. This constrains a programme's capacity to respond to the ongoing threat of insecticide resistance among EMR countries in a timely and efficient manner.

Dengue has now emerged in many countries, especially in Pakistan where escalating outbreaks underscore how major an impediment this disease is to social and economic development in the Region. Epidemics of visceral leishmaniasis in Sudan have caused high morbidity and mortality in affected communities. Likewise, major epidemics of cutaneous leishmaniasis have recently affected different parts of Afghanistan and the Syrian Arab Republic. Cutaneous leishmaniasis cases have rapidly escalated in the Syrian Arab Republic, overtaking the former epi-centre of the disease - Afghanistan. Cases have also been reported from the Islamic Republic of Iran, Pakistan and Saudi Arabia. The health of communities in diverse environments needs to be protected from vector-borne diseases in order to minimize the enormous toll on human health and cost to health systems. Reversing the trends in vector-borne diseases requires sustained commitment at all levels of society, including partners and governments, as well as sound technical support at the local level to effectively channel resources. All vectorborne-disease control programmes need to work in an integrated manner to address the challenges. Countries are urged to strengthen their disease surveillance and health information systems, develop a preparedness plan, invest in capacity building (clinical management and vector control) and implement integrated vector control with community and intersectoral participation.

Reducing mortality from vectorborne diseases to zero should be the first target for all countries of the Region in the next 10 years.

\section{References}

1. Townson $\mathrm{H}$ et al. Exploiting the potential of vector control for disease prevention. Bulletin of the World Health Organization, 2005, 12:942-947.

2. Handbook for integrated vector management. Geneva, World Health Organization, 2012 (http://whqlibdoc.who.int/publications/2012/9789241502801_eng.pdf, accessed 23 March 2014).

3. A global brief on vector-borne diseases. Geneva, World Health Organization, 2014.

4. Sustaining the drive to overcome the global impact of neglected tropical diseases - second WHO report on neglected tropical diseases. Geneva, World Health Organization, 2013.
5. Shepard DS et al. Economic impact of dengue illness in the Americas. American Journal of Tropical Medicine and Hygiene, 2011, 84(2):200-207.

6. Global plan for insecticide resistance management in malaria vectors (GPIRM). Geneva, World Health Organization, 2012.

7. Global strategic framework for integrated vector management. Geneva, World Health Organization, 2004.

8. Public health pesticide registration and management practices by WHO Member States. Report of a WHOPES survey. Geneva, World Health Organization, 2011.

9. World malaria report 2013. Geneva, World Health Organization, 2013. 